\title{
Circulation des biens et approvisionnement des villes, le raccourci par l'agriculture péri-urbaine et le rôle des femmes
}

Cheikh Ba

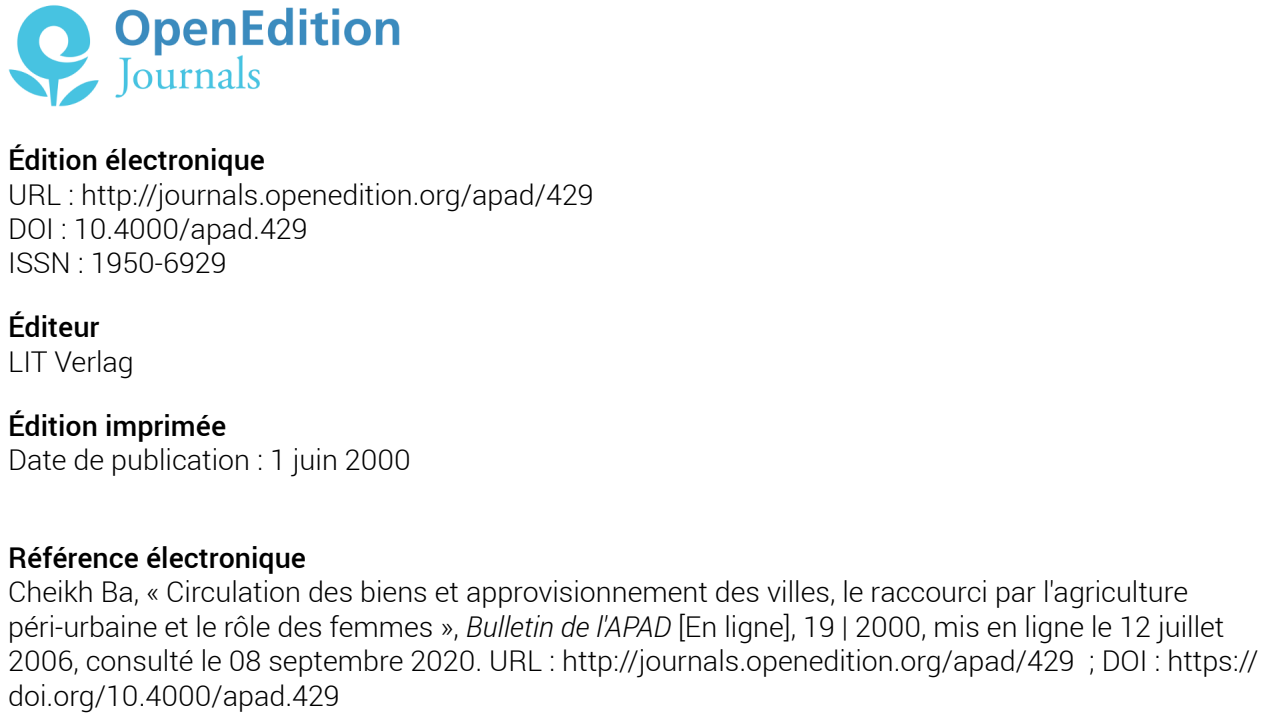

Ce document a été généré automatiquement le 8 septembre 2020

Bulletin de l'APAD 


\title{
Circulation des biens et approvisionnement des villes, le raccourci par l'agriculture péri-urbaine et le rôle des femmes
}

\author{
Cheikh Ba
}

1 Il s'agira ici d'un essai sur quelques problématiques d'ordre géographique, par rapport à la question des relations villes-campagnes en Afrique au Sud du Sahara. La circulation des ressources entre l'espace rural et les espaces urbanisés a presque toujours été perçue du point de vue particulier du ravitaillement des citadins par les ruraux. Ainsi les "zones de production" étaient opposées aux "zones de consommation". Cette approche dualiste occulte non seulement la relation d'interaction elle même, mais encore la nature de l'espace de relation dans lequel s'inscrivent les lieux, les distances, les aires, les réseaux, les infrastructures, les flux, les acteurs et tous les autres phénomènes d'accompagnement d'ordre général. La saisie de cet espace relationnel est de nos jours plus que nécessaire pour comprendre la complexité de la question de l'approvisionnement.

L'approvisionnement des villes, une question théorique

2 A la suite de la génération des monographies de villes et de villages des années 1950 et 60 , les travaux sur "l'approvisionnement des villes" se multiplient en Afrique au Sud du Sahara à partir de la décennie suivante. Ils étaient essentiellement des études géographiques de la question du ravitaillement vivrier entraîné par la récente et rapide urbanisation en Afrique tropicale. Le secteur vivrier comprenait pêle-mêle céréales, manioc, igname, taros, bananes, vin de palme, piment et autres cultures maraîchères, produits d'élevage et de pêche. La plupart de ces travaux étaient menés principalement dans le cadre du Centre d'Etude de Géographie Tropicale (CEGET) de Bordeaux, ou de thèses, de mémoires de Maîtrise de géographie ${ }^{1}$.

3 Ainsi, des enjeux ruraux (nourriture de masses rurales dominantes durant l'ère coloniale, poids décisif des cultures de rente, environnement de l'économie de traite 
tissant une armature urbaine d'escales et de ports) on est passé à de nouveaux enjeux urbains. A une nouvelle phase de croissance urbaine accélérée se sont associés des séquences de sécheresse sévères, une accentuation de la dégradation des espaces et systèmes d'exploitation, des mouvements migratoires généralisés. Tout cela compromit les capacités de ravitaillement de villes foisonnantes et croissantes par des campagnes en dépérissement.

4 La problématique de l'approvisionnement revêt dès lors un caractère stratégique de plus en plus affirmé et complexe. Gouvernements africains, anciennes métropoles et nouveaux bailleurs de fonds se retrouvent ensemble, face aux conséquences (politiques, financières et sociales) engendrées par l'insécurité alimentaire, l'inefficacité des politiques de développement, l'aide, la dépendance, les changements et mutations rapides affectant l'espace géographique dans son ensemble, et singulièrement la trame de l'espace des relations villes-campagnes. Il fallait donc lancer un certain nombre de recherches non moins stratégiques et opérationnelles, sous le couvert de l'euphémisme d'occultation de "géographie de l'alimentation" 2. Ce qui donna naissance à biens des programmes et projets financés par divers bailleurs de fonds issus de pays du Nord ou d'institutions de coopération multilatérale.

5 Au Département de géographie de l'Université de Dakar nous avons personnellement tenté un enrichissement de la problématique du ravitaillement. Partant de nos enseignements sur les relations villes-campagnes (depuis une trentaine d'années), nous avons impulsé sans financement quelconque, une série d'études et de recherches (principalement sur le Sénégal), dans le cadre de la préparation de mémoires de Maitrise et de DEA ${ }^{3}$.

6 La problématique de l'approvisionnement des villes fut alors élargie, à partir des concepts de besoin essentiel et d'espace géographique. Les rythmes de la croissance urbaine et de dépérissement des campagnes, dans un contexte politico-économique de dépendance accrue et de mal développement, ont contribué à modifier profondément la nature, la gamme, l'origine géographique, les flux et les formes d'inscription spatiale de produits et biens circulant entre la ville et la campagne. Plus que le vivrier, l'approvisionnement entre villes et campagnes embrasse les sources d'énergie, l'eau et les produits usinés. L'extraversion, la puissance croissante du marché et les conditionnalités de l'aide au développement non seulement dopent les importations de produits agricoles traditionnellement fournis aux villes par les campagnes africaines, mais encore agissent sur les données et les formes d'ordre économique et géographique de la distribution des biens et des produits.

7 En conséquence, les changements qualitatifs de l'approvisionnement des villes et des campagnes, accompagnent, voire même influencent, les changements des qualités de l'espace géographique : échelles, limites et autres lignes, aires et surfaces, distance et étendue, mobilité et autres mouvements, réflexivité et autres bases de représentation ou de symbolisme.

8 En attendant l'exploitation de la masse de documents présentés, nous pouvons faire quelques premières observations. Le concept d'organisation de la campagne par la ville (en vue d'un approvisionnement ou d'une tutelle), dans le contexte de ces études, manque toujours de prégnance. Comme toujours, depuis l'ère coloniale, les villes prélèvent sur les campagnes (biens et hommes) plus qu'elles ne les organisent. Leur manque de dynamisme propre fait le jeu des facteurs externes (marchés, échanges à longue distance, aide au développement, ajustement structure) et des facteurs 
généraux de niveau national (politiques publiques relatives à l'espace, comme les aménagements; réformisme et régionalisme politiques, croissance démographique et dégradation des systèmes et espaces de production).

9 Malgré la précarité qui frappe villes et campagnes, on remarque une vitalité considérable des phénomènes économiques, comme de la mobilité géographique. Flux, marchés foisonnants, acteurs et opérateurs de plus en plus nombreux et diversifiés (selon notamment l'occupation, le sexe, l'appartenance ethnique, voire religieuse, la résidence), transactions et prix, entre autres, s'inscrivent dans des espaces de relation extrêmement dynamiques.

Ce dynamisme est à la mesure, par exemple, de l'actualité des questions relatives à l'accessibilité géoéconomique aux marchés ruraux ou aux sources d'approvisionnement. Il concerne de même tout ce qui est relatif aux limites territoriales, au foncier, au contact espace ruraux-espaces urbanisés, ou à l'attractivité des bassins urbains et ruraux.

11 Ainsi, la problématique de l'approvisionnement s'articule de plus en plus à celle de la gestion de l'espace. Comment renforcer les capacités de l'espace rural, notamment les capacités de production, de ravitaillement des villes, de promotion de cadres de vie et de dynamisation permanente de l'équilibre ville-campagne? Comment dépasser le schéma territorial hérité de la colonisation et renforcé par les réformes politiques permanentes ? ${ }^{4}$. Un tel schéma repose sur une urbanisation et une hiérarchie urbaine de contrôle territorial plutôt que de développement régional. Ainsi les villes promues comme telles ou qui s'accroissent ne retrouvent-elles, ni en elles-mêmes, ni à travers leurs formes d'aménagement, les capacités de contrôle de leur approvisionnement, et encore moins celles de régulation de leur rapport avec les campagnes. Jusqu'où peut aller, au Sénégal comme en Afrique au Sud du Sahara, cette trame générale de l'espace des relations villes-campagnes assise sur une urbanisation artificielle et inachevée? Quelle est la pertinence du modèle urbanogénétique eurocentré, eu égard à la question de l'approvisionnement ou même d'autres questions de développement?

$\mathrm{Au}$ total, donc, la notion d'approvisionnement est un concept dynamique qui nous renvoie à l'épistémologie et à la méthodologie géographique de la relation ville-campagne.

L'approvisionnement, une question d'interrelation de nature multiscalaire

13 Le concept d'approvisionnement est de nature transversale, du double point de vue disciplinaire et interdisciplinaire. En géographie, il traverse l'ensemble des "branches" conventionnelles de la discipline: depuis l'étude des faits naturels (ressources naturelles d'origine hydrique, végétale et animale) jusqu'à celle des phénomènes dynamiques (géographie de la circulation, géographie du développement, géographie des migrations, mobilité géographique), en passant par les "branches" classiques comme la géographie rurale, la géographie urbaine, la géographie économique. Les nécessaires rapprochements avec la sociologie, la géopolitique, la cartographie et la statistique se passent de commentaires. Dès lors, cette forme particulière d'échange entre ville et campagne renvoie précisément à l'échelle, dans la mesure où c'est un phénomène humain dont les processus (économiques, spatiaux et temporels, etc.) s'inscrivent dans plusieurs niveaux géographiques de dimensions différentes. Celles-ci vont du local au mondial, en passant par le régional, l'interrégional, le national, l'international. Dans le domaine alimentaire en général, agricole en particulier, aucune filière d'approvisionnement (des villes et même de bien des campagnes) ne peut être 
analysée sans tenir compte de ce jeu de niveaux scalaires, Que ne peut-on dire sur la question céréalière! sur les cultures maraîchères ! sur le lait !

A l'échelle mondiale, d'une manière générale, le caractère, les facettes et l'intensité des relations villes-campagnes sont profondément influencés par le modèle culturel eurocentriste (consommation, goût..,), l'économie de marché, les changements affectant les systèmes de distance, et les mouvements de population.

Du point de vue géographique, le modèle culturel en question tend non seulement à brouiller les fonctions spatiales (inscription, localisation, différenciation, répartition des phénomènes humains), mais encore à écraser les "écocultures" par opacification des structures spatiales qui en portent la marque. Les études actuelles sur les terroirs africains montrent bien tout ce jeu de massacre de nombreuses variétés ou d'espèces végétales dites locales. Tout cela au nom d'une modernisation fondée sur le principe d'unicité hégémonique d'une culture de rente. Que de bassins ou d'espaces de production moderne (coton, arachide, riz...) pratiquement exclus du ravitaillement vivrier des villes en céréales (mil, fonio...), en tubercules, en produits forestiers, en lait et en viande! Que de judicieuses stratégies d'utilisation durable du sol bousculées par de douteuses technologies modernes d'aménagement de l'espace rural!

Au Sénégal que dire du "riz de la vallée", de la "tomate du Delta" ou des "violette de Galmi" (oignons) des Niayes exclus pourtant des principaux circuits d'approvisionnement des grandes villes du Sénégal, face à une énorme demande urbaine?

17 La généralisation de l'économie de marché fait de l'espace géographique dans son ensemble plus qu'une association de milieux aménagés dotés de ressources particulières (naturelles, humaines...). Elle en fait un seul système de ressources fonctionnant selon les mêmes normes et valeurs (catégories normatives, valeurs marchandes...) et la même logique (le productivisme économiste). Ainsi l'ensemble des espaces de la relation ville-campagne est gagné par le spéculation, depuis les facteurs de production jusqu'à la distribution des produits. Mais, avec la croissance urbaine et la plus grande fluidité des investissements citadins, la demande urbaine devient plus complexe. Ce n'est plus simplement le produit rural qui est sollicité, mais de plus en plus le facteur de production et la ressource. Ainsi, par exemple, la valeur foncière de la terre s'accroît très rapidement, notamment dans les espaces ruraux aménagés ou dans ceux qui sont proches des grandes villes. La capitalisation croissante tend à l'emporter sur les processus de régulation sociale traditionnelle du fonctionnement des exploitations. La privatisation, avec la complicité ou sous l'égide de l'administration ou des collectivités locales, érode les patrimoines, les réserves, les aires protégées. Les communautés traditionnelles sont gagnées par les groupements d'intérêt économique (GIE).

18 Ce sont là autant de processus et de changements qui affectent les espaces de production, les formes d'exploitation rurale, les formes et la dynamique de l'approvisionnement des villes. Ce sont même autant de données économiques émergentes qui contribuent à la complexité des stratégies relatives à l'espace géographique de relation ville-campagne: utilisation productive du sol, accès à la ressource (foncière, forestière, halieutique..,), sécurisation foncière, territorialisation des réseaux commerciaux et des filières d'approvisionnement.

Dans un tel contexte, au niveau africain, les espaces de production, de consommation et de relation. ruraux comme urbains, sont entraînés dans ce réseau mondial structuré 
par le capital, les stratégies des bailleurs de fonds et la coopération internationale centralisée ou décentralisée.

Les changements affectent de façon lisible les niveaux régionaux et locaux d'organisation des relations d'échange entre villes et campagnes. Les bases matérielles de l'approvisionnement (spéculations, exploitations, équipements, infrastructures et outils) s'en retrouvent fortement dépendantes d'un "extérieur" sans localisation géographique apparente. L'exportation des produits ruraux de meilleure qualité (riz, production maraîchères et fruitières, poisson...) ralentit plus ou moins la structuration des systèmes régionaux et locaux d'approvisionnement. Le marché induit un système de ravitaillement sélectif et inégalitaire au ni veau national.

Une telle dynamique des relations d'échange entre villes et campagnes, impulsée par ces phénomènes d'ordre culturel et économique, se consolide de nos jours, sous le double impact des changements affectant les systèmes de distance d'une part, et de migrations, d'autre part. La nouvelle série des mémoires que nous avons consacrée aux rapports villes-campagnes à longue distance ${ }^{5}$ apporte déjà un bon début d'éclairage d'une facette émergente de la problématique des relations villes-campagnes.

Les changements récents dans les mouvements migratoires intercontinentaux (Afrique-Europe, Afrique-Amérique) induisent progressivement des transformations significatives. Celles-ci concernent non seulement les flux d'échange (transfert monétaires, biens de consommation, marchandises de récupération du genre "venant de France"...), mais encore les paysages géographiques des vieux bassins de production agricole et la structure de l'approvisionnement des villes régionales et de nombreux villages.

23 Au total, à l'échelle mondiale la dimension géographique de l'approvisionnement des villes comme des campagnes procède d'un niveau d'organisation profondément globalisé et "virtualisé". Sa lecture directe est non moins biaisée par la massification et la diversification des courants Nord-Sud.

24 A l'échelle régionale, bien évidemment, la pertinence de toute analyse de la relation ville-campagne, en général et de l'approvisionnement des villes, en particulier, dépend du niveau de région considérée. En Afrique occidentale, par exemple, le "cachet" régional des relations villes-campagnes procède de l'empreinte coloniale. Echelle géographique et échelle temporelle de la question de l'approvisionnement sont indissociables. Nombreuses sont les formes de latence du marquage colonialiste de l'espace. Il en est de même de l'extraversion des productions et des échanges. Le dualisme ville-campagne, globalement, fonctionne dans tous les pays. Le semis urbain dominant littoral à sublittoral, renforce le maintien des courants traditionnels de migration et les flux économiques des savanes vers les côtes.

Comme souligné plus haut, c'est par conséquent l'économie de traite qui a été à l'origine de la trame de l'espace des relations villes-campagnes en Afrique occidentale, trame tissée par les escales (sur fleuves, rails, routes et autres pistes de ramassage), les ports, les bassins de production et de drainage nommés à partir de la culture dominante (arachide, café, coton, riz...). C'est d'elle que procèdent toutes les traditions relatives à l'approvisionnement: tradition d'un système économico-spatial nettement partagé entre escales et campagnes ; tradition migratoire de transfert de produits et de main d' œuvre vers les foyers et centres actifs. 
26 Ainsi devint-elle à la fois la condition primordiale, le facteur et la forme des relations villes-campagnes. La dualité du secteur approvisionnement (le produit vivrier suivait le produit de rente vers l'escale), l'ordonnancement des relations entre l'hinterland provincial et les escales ${ }^{6}$, malgré l'accroissement postérieur des flux démographiques et économiques responsables de l'urbanisation massive récente, marquent encore le développement spatial. Sous ce rapport la dynamique urbaine impulsée par l'administration coloniale et son système de traite a fait de l'escale un espace de transaction, de migration et de contrôle territorial qui s'est conservé dans toute l'ex-AOF. Aucune des solutions de reconversion d'une telle économie ou de maitrise d'une telle croissance urbaine n'a pu, jusqu'à nos jours, résorber ce schéma économico-spatial d'organisation de l'approvisionnement des villes modernes.

27 A l'échelle locale, c'est-à-dire à grande échelle, du village à la petite ville et aux unités territoriales inférieures (cantons, communauté rurale, arrondissement ou département... selon les pays africains considérés), les relations d'échange évoluent ou accusent des mutations plus rapides. Elles sont géographiquement plus significatives d'une certaine recomposition de l'espace rural.

Les formes les plus notables en sont la revitalisation du marché intérieur rural et les stratégies d'autonomisation (développement local par le biais de la communalisation rurale, de la régionalisation politique, de la promotion d'un encadrement non gouvernemental, enfin d'une connexion plus directe avec l'échelle mondiale à travers projets, transferts et coopération). Si bien que dans des secteurs ruraux, on note soit une certaine ruralité influençant l'économie urbaine locale (en la dynamisant plus ou moins nettement) ${ }^{7}$, soit un contrôle assez poussé de l'espace rural par les gros villages-centres autonomisés par la communalisation rurale ${ }^{8}$.

En conclusion, il convient de noter toute la pertinence de l'analyse multiscalaire de la réalité de la relation ville-campagne en général et de la complexité de l'inscription géographique des processus et formes de l'approvisionnement. Selon les échelles, leur imbrication rend parfois opaque la dualité des termes de cette relation. Mais en Afrique au Sud du Sahara, contrairement à l'Europe, par exemple, où l'urbanisation est quasi achevée dans les grandes régions économiques, où les villes sont réellement autochtones et où l'uniformisation normative a gommé et continue de gommer bien des personnalités géographiques, une telle relation est réelle et multiforme. Elle en marque toute la géographie humaine. Le "champ spectral" rural-urbain y est extérieurement vivant. Selon les niveaux d'analyse considérés, la problématisation des émergences, des diversités, des nuances, de la différence, voire des paradoxes, implique plus que jamais la géographie.

Conclusion

30 La question de l'approvisionnement est une question majeure. L'urbanisation massive en Afrique au Sud du Sahara, d'une part, la dégradation avancée des systèmes de ressources rurales, d'autre part, la place au cœur des interactions villes-campagnes. Elle entraîne avec elle, en les impliquant davantage, toutes les autres formes d'échange et de circulation entre ces deux termes géographiques d'organisation d'une relation très dynamique de nos jours.

31 Le concept d'approvisionnement est de ce fait fort révélateur de la complexité et de la prégnance du concept même de relation ville-campagne, au moins à quatre niveaux d'analyse. Le premier concerne la dialectique situations-dynamiques, sur le plan humain, en général, et géoéconomique, en particulier. Les situations plus ou moins 
stables, liées par exemple aux filières de ravitaillement des villes (animaux sur pied, poisson, fruits tout venant, produits forestiers...), à des zones géographiques (savane-forêt, à partir de bassins de production, marchés ruraux...), aux saisons, à des groupes ethnoterritoriaux, sont de plus en plus déstabilisés par l'urbanisation. Comme elles le sont aussi par l'application désordonnée de politiques publiques relatives à l'espace (réforme territoriale, aménagements, importations massives liées à la sécurisation alimentaire...).

32 Le deuxième niveau renvoie à la compréhension des changements affectant le jeu des facteurs de la relation ville-campagne. Parmi ceux-ci figurent les ressources (terres, eaux, forêts, mines...), leur accessibilité et leur mobilisation, les espaces de production et de distribution, les infrastructures, la démographie, la mobilité géographique...

Le troisième niveau concerne la compréhension ou l'intégration méthodologique des variables émergentes. Parmi elles, on peut retenir les processus de normalisation des besoins essentiels des villes et des campagnes, la rapide urbanisation des campagnes, la ruralisation de bien des grandes villes, la recomposition rurale portée par le développement du marché intérieur (notamment à travers les vastes bassins de culture de rente hérités de l'exploitation coloniale).

Le quatrième niveau est celui de l'affrontement des difficultés d'ordre sémantique par le chercheur. La question de l'approvisionnement, comme soulignée plus haut, est transversale. Les décalages, les disparités ou les divergences entre les points de vue et les méthodes d'une discipline à l'autre, entretiennent encore un large flou conceptuel. Comment se dessine le champ conceptuel de la relation ville-campagne? Celui des termes d'approvisionnement, de vivrier marchand, de migration, d'exode rural, de territoire, de ville...?

Ainsi se mesure la pertinence actuelle des phénomènes et procédures géographiques dans le champ descriptif et explicatif de la relation ville-campagne, en général, et dans la problématique de l'approvisionnement en particulier.

En plus de la nécessaire démarche multiscalaire soulignée plus haut, il convient d'insister sur la perspective de l'aménagement ou de la gestion de l'espace. Car le fonctionnement global des relations villes-campagnes dépend de la régulation intégrée de trois niveaux solidaires d'organisation de l'espace géographique : grandeur, ordre et territorialité.

37 En somme, on peut dire que la prise en compte du niveau "grandeur" permet de situer, entre autres, les rapports quantitatifs entre espaces urbains et espace ruraux. La démarche quantitative éclaire les procédures relatives aux phénomènes de croissance ou de décroissance (démographiques, spatiales, matérielles), à l'inscription spatiale du "binôme" offre-demande, aux formes et rythmes de consommation d'espace et aux enjeux fonciers et financiers qu'ils portent, enfin aux flux (biens, marchandises, hommes, services).

38 Le niveau "ordre", de nature plutôt géocartographique, met en exergue la structure de l'inscription spatiale des situations et leurs processus de transformation. Il montre de plus comment s'ordonnent ou se "régulent" dans l'espace non seulement les divers flux et relais, mais encore les différentes stratégies spatiales (pérennisation de situations, de réseaux, de promotion de filières d'amont en aval). Par conséquent, l'articulation des qualités relatives de l'espace géographique (échelle, distance, aires, surfaces, limites, 
mobilité...) et des phénomènes dynamiques représente une procédure méthodologique essentielle.

Le niveau "territorialité" articule en particulier formes et stratégies d'appropriation, d'utilisation ou de contrôle (sol, ressources, aménagements...), représentations de pouvoirs sur l'espace (terres, ressources, réseaux, circuits...), enfin lieux d'enjeux (fonciers, territoriaux, accessibilité...). C'est donc un niveau d'intégration dynamique.

C'est aussi le niveau qui porte le plus les variables émergentes qui modifient de nos jours les formes de relations villes-campagnes, le contenu de l'approvisionnement, les systèmes spatiaux plus ou moins individualisés par les flux, les marchés, les différentes catégories de ville, enfin les infrastructures. Ces variables vont du géographique proprement dit (principalement le rapport de l'homme à l'espace réglant les stratégies d'établissement, d'utilisation du sol ou des ressources), ou politique (réformisme d'État en Afrique ou désengagement de l'État des circuits de production et de distribution des denrées, de l'eau, des sources d'énergies, de l'encadrement rural...). Elles embrassent aussi des données d'ordre culturel (identité des aires et des groupes humains producteurs, commerçants ou transporteurs, diffusion des modèles, normes, goûts), social (travail salarié et "monétarisation" des services et assistance traditionnels...) et économique (enjeux de développement rural, insertion des "produits" humains et financiers de la migration dans l'approvisionnement; stratégies relatives à la "crise" ; spéculations conquérantes..).

\section{BIBLIOGRAPHIE}

CEGET 1972. Dix études sur l'approvisionnement des villes. Document $\mathrm{n}^{\circ} 27$.

Diaoune A., 1998. De nouvelles communes en zone rurale : enjeux et perspectives de l'érection en commune à travers l'exemple de Poût, Mémoire de DEA, Dakar : Département de Géographie, UCAD.

Dieng C.N., 1999. Relation ville-campagne : processus de domination et de contrôle de l'espace par la ville : l'exemple de Mboro, Mémoire de DEA, Dakar : Département de Géographie, UCAD.

Kane ?, 1999. Marchés ruraux périodiques et fait urbain dans le Bassin Arachidier Oriental. Mémoire de DEA, Dakar : Département de Géographie, UCAD.

Kane Mbaye F., 1999. Impact de la migration de retour des modous ruraux de New York dans leur zone de départ, Mémoire de DEA, Département de Géographie, Dakar : UCAD.

I.E.D.E.S. 1976., "L'approvisionnement des villes dans les États africains et malgaches (Horizons 1985)". Revue Tiers-Monde, XVII, 67 : 751-760.

Mboup B., 1999. Migrations internationales et développement local à Kébémer. Mémoire de DEA, Département de Géographie, Dakar : UCAD.

Mondjanagni C.A., 1977. Campagnes et villes au Sud de la République Populaire du Bénin. Paris : Mouton. 
Ndiaye M.B., 1997. De la culture vivrière au vivrier marchand : l'espace de mutation fonctionnelle d'une céréale (mil) dans le Département de Kaolack. Mémoire de DEA, Dakar : Département de Géographie, UCAD.

Nyassogbo G.K., 1975. Contribution à l'étude des relations villes-campagnes dans la Région des Plateaux (Togo). Toulouse, doctorat $3^{\mathrm{e}}$ cycle.

\section{NOTES}

1.Colloque international sur la Croissance urbaine en Afrique Noire et en Madagascar, Bordeaux-Talence, 1972, CNRS-CEGET.

2.Laquelle fera naître pendant les décennies 80 et 90 d'autres études relatives à la sécurité alimentaire, à la nourriture des villes, au vivrier marchand..., dans le champ des sciences sociales.

3.Pas moins d'une centaine de mémoires dirigés personnellement ou en collaboration avec d'autres collègues sur le thème des relations villes-campagnes proprement dit, sur la problématique de l'approvisionnement par rapport à la formation du marché intérieur rural (marchés hebdomadaires, transports, services...), aux réformes foncières et territoriales (communautés rurales, loi sur le domaine national, lois de décentralisation), à la gestion des ressources naturelles (exploitation forestière, alimentation en eau et en énergie...), à la migration et aux problèmes de population, au péri urbain et à l'extension des villes sur les terres rurales, à l'urbanisation des campagnes. Naturellement cette contribution-ci ne sied pas à l'établissement d'une quelconque liste bibliographique.

4.Voir les lois de réforme administrative ou de communalisation rurale et urbaine promulguées entre 1972 et 1996 au Sénégal. On sait aussi que pratiquement tous les pays africains issus de la colonisation française sont en train de faire la même chose. 5.Voir par exemple Kane Mbaye (1999).

6.Au Sénégal. par exemple, les Wolof ont symbolisé cette structure territoriale et cette dynamique économique en opposant le haut-pays (kow-ga) et les escales (teeru-ya) ou localités (dëk) urbanisées (taax-ya).

7.Cf Kane (1999) et Ndiaye (1997).

8.Cf Dieng (1999) et Diaoune (1998). 